\title{
Wind noise spectra in small Reynolds number turbulent flows
}

\author{
Sipei Zhao ${ }^{\text {a) }}$ \\ School and Engineering, RMIT University, Melbourne, Australia \\ Eva Cheng \\ Centre for Audio, Acoustics and Vibration, Faculty of Engineering and IT, University of Technology Sydney, \\ Sydney, Australia \\ Xiaojun Qiu and lan Burnett \\ Centre for Audio, Acoustics and Vibration, Faculty of Engineering and IT, University of Technology Sydney, \\ Sydney, Australia \\ Jacob Chia-chun Liu \\ Department of Water Resources and Environmental Engineering, Tamkang University, Taiwan
}

(Received 1 June 2017; revised 18 October 2017; accepted 3 November 2017; published online 22 November 2017)

Wind noise spectra caused by wind from fans in indoor environments have been found to be different from those measured in outdoor atmospheric conditions. Although many models have been developed to predict outdoor wind noise spectra under the assumption of large Reynolds number [Zhao, Cheng, Qiu, Burnett, and Liu (2016). J. Acoust. Soc. Am. 140, 4178-4182, and the references therein], they cannot be applied directly to the indoor situations because the Reynolds number of wind from fans in indoor environments is usually much smaller than that experienced in atmospheric turbulence. This paper proposes a pressure structure function model that combines the energy-containing and dissipation ranges so that the pressure spectrum for small Reynolds number turbulent flows can be calculated. The proposed pressure structure function model is validated with the experimental results in the literature, and then the obtained pressure spectrum is verified with the numerical simulation and experiment results. It is demonstrated that the pressure spectrum obtained from the proposed pressure structure function model can be utilized to estimate wind noise spectra caused by turbulent flows with small Reynolds numbers.

(C) 2017 Acoustical Society of America. https://doi.org/10.1121/1.5012740

[ATW]

Pages: $3227-3233$

\section{INTRODUCTION}

Wind noise is the pressure fluctuations caused by turbulence around the microphone, which consists of the intrinsic turbulence in the incoming flow and/or the wake generated by the microphone, of which the former is dominant in windy conditions (Morgan and Raspet, 1992). There has been wide research on the pressure spectra in turbulent flows and outdoor measurements of the wind noise spectra in the past few decades (Hill and Wilczak, 1995; Raspet et al., 2006). It is found that the Reynolds number based on the Taylor microscale varies from 4250 to 19500 in atmospheric turbulence (Pearson and Antonia, 2001), and a pressure spectrum model has been proposed by the authors to predict the wind noise spectra in such large Reynolds number turbulent flows (Zhao et al., 2016). This paper investigates the wind noise spectra in small Reynolds number turbulent flows such as the wind from fans in indoor environments.

Turbulence spectra can be divided into three regions: the energy-containing range where the eddy size is comparable to the mean flow, the inertial range where the inertial effect dominates the eddy motion, and the dissipation range where the kinetic energy is dissipated to heat by viscous effect (Pope, 2000). Previous theories focused on the inertial

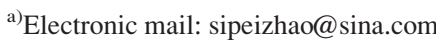

range where the pressure spectrum was found to vary as $k^{-7 / 3}$ ( $k$ is the wavenumber) (Batchelor, 1951; Hill and Wilczak, 1995), and the theories have been utilized to predict the wind noise spectrum in the inertial range from the measured velocity spectrum in atmosphere (Raspet et al., 2006).

Raspet et al. (2008) extended the pressure spectra to the low frequency region of the energy-containing range based on the spectral model developed by George et al. (1984). The infrasonic wind noise spectrum was measured under both pine and deciduous tree canopies where the turbulenceshear interaction is found to correspond to the low frequency peak in the wind noise spectrum, while the turbulence-turbulence interaction pressure with the $-7 / 3$ power law dominates the higher frequency region in the inertial range (Raspet and Webster, 2015; Webster and Raspet, 2015). Recently, we proposed a pressure structure function model that incorporates both the inertial and dissipation ranges so that the pressure spectrum was extended to the dissipation range (Zhao et al., 2016). The obtained pressure spectrum follows the $-7 / 3$ power law in the inertial range and falls off rapidly in the dissipation range in the higher frequency region. The model was validated with the wind noise spectrum measured in an outdoor car park.

In summary, the existing research was devoted to the inertial range and the extension to lower frequency (Raspet et al., 2008) and higher frequency regions (Zhao et al., 
2016), which were validated with wind noise spectra measured in outdoor environments. In addition to the above outdoor investigations, wind noise has also been measured in indoor environments such as wind tunnels. Recent measurements of wind noise in a small anechoic wind tunnel showed that the noise spectrum does not change significantly in the lower frequency region but decays much faster than the $-7 / 3$ power law in the higher frequency region, which is inconsistent with wind noise measured outdoor (Alamshah et al., 2015; Wang et al., 2012). This may be due to the smaller Reynolds number of the wind tunnel flows than those found in atmospheric flows.

Recent numerical simulation and experiment results also showed that the inertial range with the $-7 / 3$ power law cannot be observed in small Reynolds number flows but only exists in the turbulent flows with sufficiently large Reynolds numbers (Gotoh and Fukayama, 2001; Tsuji and Ishihara, 2003). The numerical simulations by Gotoh and Fukayama (2001) showed that the $-7 / 3$ power law can only be observed when the Taylor microscale Reynolds number is larger than 284, while the experiment results in wind tunnels by Tsuji and Ishihara (2003) confirmed the $-7 / 3$ power law when the Taylor microscale Reynolds number was larger than 600. Meldi and Sagaut (2013) argued that a Taylor microscale Reynolds number larger than $10^{4}$ is necessary to observe the $-7 / 3$ power law in the pressure spectrum.

Although wind noise spectra measured in outdoor atmospheric turbulence with a sufficiently large Reynolds number can be described with the existing model in the inertial and dissipation ranges, no theory exists to predict the pressure spectrum in small Reynolds number turbulent flows where the inertial range is absent. To better understand the wind noise measured in indoor environments with small Reynolds numbers, such as that under fans or air conditioner outlets, this paper proposes a pressure structure function model that incorporates the energy-containing and dissipation ranges to predict the pressure spectrum for small Reynolds number turbulent flows. Existing literature data and measurement results from indoor fan tests are used to validate the proposed pressure structure function model and the obtained pressure spectrum.

\section{THEORY}

The pressure structure function describes the spatial relationship between pressures at two locations and is defined as (Hill and Wilczak, 1995)

$$
D_{p}(r)=\frac{1}{\rho^{2}}\left\langle(p(x)-p(x+r))^{2}\right\rangle,
$$

where $p(x)$ is the pressure at position $x, r$ is the separation distance between two spatial locations, and $\langle\cdot\rangle$ denotes the ensemble average. Eddies with dimensions much larger than the separation distance $r$ do not affect the pressure difference significantly; thus the pressure structure function is mainly determined by eddies with dimensions comparable to $r$ (Tatarski, 1961).

Hill and Wilczak (1995) proposed a theoretical model to relate the pressure structure function to the fourth-order velocity structure function from the Poisson equation, and obtained an asymptotic form pressure structure function in the energy-containing range, the inertial range and the dissipation range. In the energy-containing range with sufficiently large separation distance, the pressure structure function is twice the pressure variance for the homogeneous and isotropic turbulence, as given in Eq. (2). In the inertial range where the separation distance $r$ is much smaller than the size of the largest eddy but much larger than the size of the smallest eddy, the eddy motions are solely determined by the energy dissipation rate. The pressure structure function in this range increases with the separation distance according to an exponent of $4 / 3$, which can be written in a universal form as in Eq. (3). In the dissipation range where the separation distance $r$ is comparable to the Kolmogorov scale $\eta$, the square of the pressure difference at two spatial locations increases with the squared separation distance which is faster than that in the inertial range. Equation (4) gives the pressure structure function in this range:

$$
\begin{aligned}
& D_{p}(r) \approx 2 \sigma_{P}^{2}=2 \frac{\left\langle P^{2}\right\rangle}{\rho^{2}}, \\
& D_{p}(r) \approx C_{p} \varepsilon^{4 / 3} r^{4 / 3}, \\
& D_{p}(r) \approx \frac{1}{3} A r^{2},
\end{aligned}
$$

where the pressure variance $\sigma_{P}^{2}$ is a constant for a certain turbulent flow, $C_{p}$ is a constant, $\varepsilon$ is the energy dissipation rate, $A=\int_{0}^{\infty} r^{-3} D_{1111}(r) d r$ is independent of $r$ (Ould-Rouis et al., 1996). $D_{1111}(r)=\left\langle(u(x)-u(x+r))^{4}\right\rangle$ is the fourth order longitudinal velocity structure function, $u$ is the longitudinal velocity and the subscript number 1 denotes the longitudinal direction (Hill and Wilczak, 1995).

The pressure spectrum can be calculated from the pressure structure function by (Lohse and Muller-Groeling, 1995)

$$
P(k)=-\frac{1}{2 \pi} \int_{0}^{\infty} D_{p}(r) \sin (k r) k r d r,
$$

where $k$ is the wavenumber. In the previous research focused on the inertial range, Eq. (3) was substituted into Eq. (5) to obtain the pressure spectrum that varies as $k^{-7 / 3}$ in the inertial range (Hill and Wilczak, 1995). Zhao et al. (2016) proposed to combine the pressure structure function in the inertial range in Eq. (3) and the dissipation range in Eq. (4) so that the pressure spectrum can be extended to the dissipation range. However, this model is only valid for turbulent flows with sufficiently large Reynolds numbers such that the inertial range always exists.

For turbulent flows with small Reynolds numbers, there is no inertial range (Gotoh and Fukayama, 2001; Tsuji and Ishihara, 2003). To accurately describe the pressure spectrum in such flows, this paper proposes an alternative pressure structure function model that incorporates the energy-containing range in Eq. (2) and the dissipation range in Eq. (4), namely,

$$
D_{p}(r) \approx \frac{1}{3} \frac{A r^{2}}{\left[1+\left(r / r_{\mathrm{L}}\right)^{2}\right]},
$$

where $r_{\mathrm{L}}=\left(6{\sigma_{P}}^{2} / A\right)^{1 / 2}$ denotes the transition from the dissipation range to the energy-containing range, and can be 
obtained by equating Eqs. (2)-(4). For $r \gg r_{\mathrm{L}}$, Eq. (6) approaches Eq. (2) in the energy-containing range while for $r \ll r_{\mathrm{L}}$, Eq. (6) approaches Eq. (4) in the dissipation range.

Substitute Eq. (6) into Eq. (5), the pressure spectrum can be obtained:

$$
P(k)=\frac{A r_{\mathrm{L}}^{3}}{6 \pi} e^{-k r_{\mathrm{L}}} .
$$

The transition between the energy-containing range and the dissipation range occurs at $1 / r_{\mathrm{L}}$ in the wavenumber space. In the energy-containing range $\left(k r_{\mathrm{L}} \ll 1\right)$, the exponential term approaches to 1 so the proposed pressure spectrum model approaches a constant and does not vary with the wavenumber (or equivalently frequency), which is consistent with the measurement results of the wind noise spectra in a small anechoic wind tunnel (Alamshah et al., 2015; Wang et al., 2012). In the dissipation range $\left(k r_{\mathrm{L}} \gg 1\right)$, the pressure spectrum falls off rapidly as the exponential decay, which is consistent with the dissipation range spectrum in the previous model developed by Zhao et al. (2016). The value of $r_{\mathrm{L}}$ depends on the constant $A$ and the pressure variance $\sigma_{P}{ }^{2}$ by $r_{\mathrm{L}}=\left(6 \sigma_{P}{ }^{2} / A\right)^{1 / 2}$. The pressure variance can be calculated from the measured pressure fluctuations. The constant $A$ is determined by the fourth order longitudinal velocity structure function. Therefore, the calculation of the exact value of the constant $A$ needs accurate measurement of the longitudinal velocity at two spatial locations with various separation distances.

The main contribution of this paper is the proposed pressure structure function model in Eq. (6) and the derivation of the pressure spectrum in Eq. (7), which can be used to predict the pressure spectra in turbulent flows with small Reynolds numbers, such as the wind noise spectra caused by wind from fans and those measured in small anechoic wind tunnels. This is different from previous models for outdoor wind noise spectra that focus on the inertial range (Raspet et al., 2006), or the combination of the inertial range and the dissipation range (Zhao et al., 2016), both of which assume that the Reynolds number is so large that the inertial range always exists.

The physical meaning of the obtained turbulent pressure spectrum for small Reynolds number turbulent flows can be explained based on the energy cascade theory (Pope, 2000). In turbulent flows, the largest eddies contain most of the kinetic energy whereas the smallest eddies convert the kinetic energy to thermal energy via the viscous dissipation. The intermediate size eddies in between are responsible for the kinetic energy transfer from the largest eddies to the smallest eddies, which is called the inertial range. The width of the inertial range depends on the difference between the size of the largest and smallest eddies. For the turbulent flows with very large Reynolds number, such as the atmospheric turbulence, the largest eddies in the energycontaining range can be in hundreds of meters while the smallest eddies in the dissipation range is the order of millimeters, therefore a wide inertial range can be observed in the pressure spectrum (Zhao et al., 2016). However, for the turbulent flows with small Reynolds number, such as the wind from fans used in this paper, the largest eddies is the order of centimeters (determined by the fan blade length $\sim 10 \mathrm{~cm}$ ), which is much smaller than the atmospheric turbulence. In this case, the kinetic energy transfer to the smallest eddies and is dissipated into heat quickly, so there is no inertial range with the $k^{-7 / 3}$ law in the pressure spectrum.

The limitation of the proposed model is that it is only valid for turbulent flows with small Reynolds numbers where the inertial range is absent, and the effect of the Reynolds number is not explicitly expressed in the model. The proposed pressure structure function model in Eq. (6) and the obtained pressure spectrum in Eq. (7) will be validated with both existing numerical and experimental data from the literature as well as our own simple indoor fan test results in Sec. III.

\section{VALIDATIONS AND DISCUSSIONS}

The proposed pressure structure function model for small Reynolds number turbulent flows in Eq. (6) is compared here with that from the experiment results by OuldRouis et al. (1996) in Fig. 1. The values of $\eta$ and $r_{\mathrm{L}}$ were not given in the literature with the experiment results, so the proposed pressure structure function model in Eq. (6) was fitted to the experiment results in Fig. 1 with $r_{\mathrm{L}}=30 \eta$. It can be observed from Fig. 1 that for small Reynolds number turbulent flows, existing theories that assume $D_{p}(r) \sim r^{4 / 3}$ cannot describe the pressure structure function, whereas the proposed pressure structure function model in Eq. (6) shows good agreement in both the dissipation range with $D_{p}(r) \sim r^{2}$ for small separations and in the energy-containing range where $D_{p}(r)$ tends to constant for large separations. It is noteworthy that there is no inertial range with $D_{p}(r) \sim r^{4 / 3}$ in the experiment results because the Reynolds number is small.

The pressure spectrum obtained from the proposed model in Eq. (7) is compared with the existing direct numerical simulation (DNS) results in Fig. 2 (Gotoh and Fukayama, 2001; Kim and Antonia, 1993; Pumir, 1994). The pressure spectrum refers to the power spectral density of the pressure fluctuation and has a unit of $\mathrm{Pa}^{2} / \mathrm{Hz}$ in this paper. The pressure spectrum normalized by the energy dissipation rate $\varepsilon$

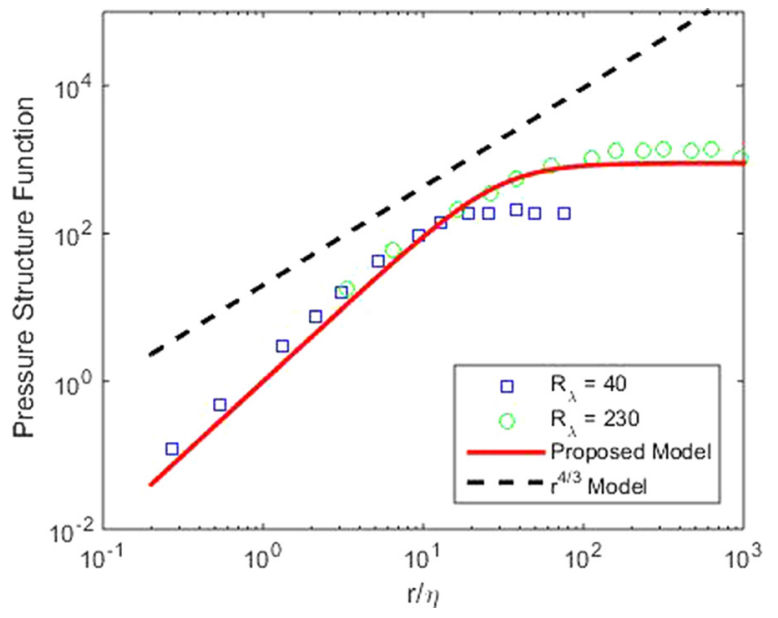

FIG. 1. (Color online) Comparison of the proposed pressure structure function model in Eq. (6) with the experiment results from Ould-Rouis et al. (1996). The abscissa is normalized with the Kolmogorov scale $\eta$. 


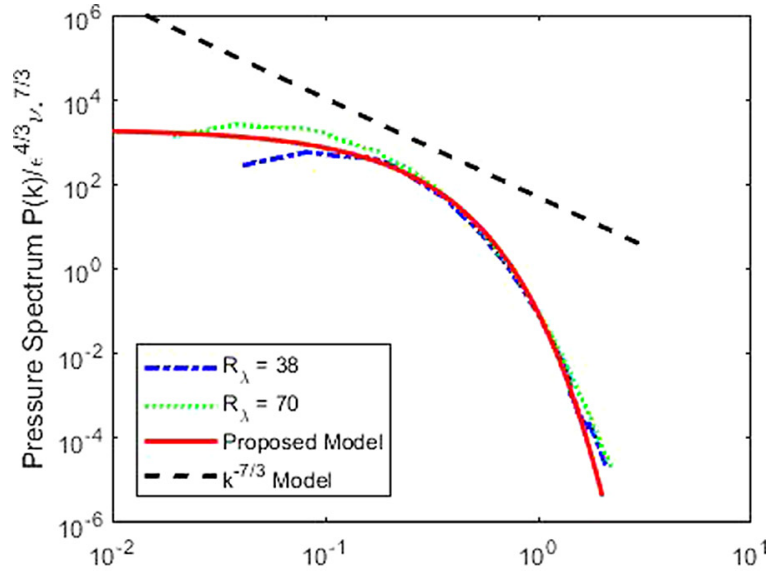

(a)

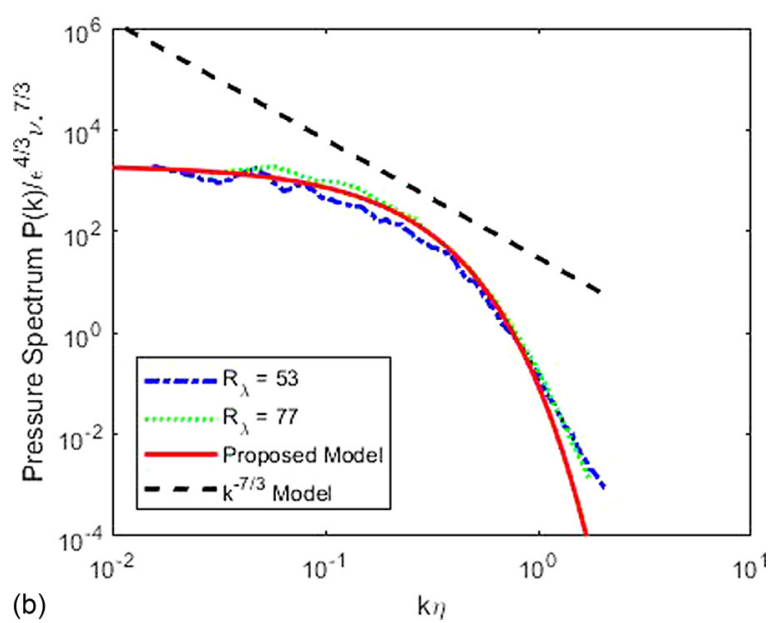

FIG. 2. (Color online) Comparison of the pressure spectrum obtained with the proposed model in Eq. (7) with the existing DNS simulation results from (a) Gotoh and Fukayama (2001), and (b) Kim and Antonia (1993) and Pumir (1994). The abscissa is normalized with the Kolmogorov scale $\eta$.

and the air viscosity $\nu$, i.e., $P(k) / \varepsilon^{4 / 3} \nu^{-7 / 3}$, is read from the figures in the source literature, as shown in Fig. 2. The values of the energy dissipation rate, the Kolmogorov scale $\eta$ and the transition constant $r_{\mathrm{L}}$ were not given in the literature with the simulation results, so the pressure spectrum model in Eq. (7) was fitted to the simulation results in Fig. 2 with $r_{\mathrm{L}}=10 \eta$. Figure 2 shows that the simulated pressure spectrum tends to be constant in the lower frequency region while it decays rapidly in the higher frequency region. The pressure spectrum obtained from the proposed model in Eq. (7) agrees well with the simulation results, where the lower frequency region corresponds to the energy-containing range and the higher frequency region corresponds to the dissipation range. There is no inertial range in the simulation results due to the small Reynolds number, so the traditional $k^{-7 / 3}$ model is not valid in this case. The pressure spectra in the turbulent flows with small Reynolds numbers are predicted by the pressure spectrum obtained from the proposed pressure structure function model in Eq. (6), which cannot be obtained with the traditional asymptotic form pressure structure function.

To further validate the pressure spectrum obtained from the proposed pressure structure function model, the wind noise spectra from a fan were measured in the SIAL sound pod at RMIT University. The SIAL sound pod is a small room where the walls and floor are finished with sound absorptive material. The fan and the microphone were about $0.8 \mathrm{~m}$ above the floor, with a separation distance of $0.5 \mathrm{~m}$. The diagram and the photo of the experimental setup are shown in Fig. 3. The wind noise was measured with a B\&K type 4189 prepolarized free field $\frac{1}{2}$ in. microphone whose frequency response is $2.8 \mathrm{~Hz}-20 \mathrm{kHz}$, and a G.R.A.S Type $40 \mathrm{BF} \frac{1}{4}$ in. free field microphone, whose frequency response is $10 \mathrm{~Hz}-40 \mathrm{kHz}$, respectively. The $\frac{1}{2}$ in. microphone was connected to the B\&K type 2270 Analyser via a B\&K Type ZC 0032 Preamplifier. The system was calibrated with a B\&K type 4231 calibrator. The $\frac{1}{4}$ in. microphone was connected to a ZOOM H6 recorder via a G.R.A.S. Type 26AC preamplifier and a G.R.A.S. Type 12AA power module. The system was calibrated with a G.R.A.S. Type 42AA Pistonphone. The mean wind speed was measure with a DIGITECH QM1646 Hand-held Anemometer.

To confirm the measured noise spectra is caused by wind from the fan when the microphone is placed inside the air flow, the $\frac{1}{2}$ in. microphone was placed in front of the fan (inside the flow) and behind the fan (outside the flow) to measure the wind and mechanical noise of the fan, respectively. In the experiment, the fan ran at its highest speed and the average wind velocity around the microphone was about $4.2 \mathrm{~m} / \mathrm{s}$. The Reynolds number based on the dimension of the fan can be estimated as $\operatorname{Re}=U D / \nu=2.8 \times 10^{4}(U$ is the wind velocity, $D=0.1 \mathrm{~m}$ is the length of the fan blade and $\nu$ is the air kinematic viscosity). The Taylor Reynolds number $\operatorname{Re}_{\lambda}$ is proportional to the square root of the Reynolds number, i.e., $\operatorname{Re}_{\lambda} \approx(20 \operatorname{Re} / 3)^{1 / 2}=432$ (Pope, 2000). The wind noise spectra were measured for $30 \mathrm{~s}$ with the $\frac{1}{2}$ in. and $\frac{1}{4}$ in. microphones, respectively. The distance between the fan and the $\frac{1}{2}$ in. microphone was $0.5 \mathrm{~m}$ in both cases.

The measurement results in Fig. 4 indicate that the overall noise level is much lower when the $\frac{1}{2}$ in. microphone is outside the flow, hence the measurement results with the microphone placed inside the air flow were primarily due to the turbulence in wind from the fan. The vertical axis in Fig. 4 is the sound pressure level (SPL) in $\mathrm{dB}$ scale with a reference pressure of $20 \mu \mathrm{Pa}$. Figure 4 also shows the wind noise spectra measured with the $\frac{1}{2}$ in. microphone parallel with the air flow direction, which is almost the same as that measured with the microphone perpendicular to the air flow direction. The following results were all measured with the microphone perpendicular to the air flow.

The measurement results with the $\frac{1}{2}$ in. and $\frac{1}{4}$ in. microphones placed inside and perpendicular to the air flow are compared with the proposed model and the conventional $k^{-7 / 3}$ model in Fig. 5. The wind noise spectra are measured at wind speeds $U=1.0 \mathrm{~m} / \mathrm{s}$ and $U=3.8 \mathrm{~m} / \mathrm{s}$, which correspond to the Taylor microscale Reynolds number of 210 and 410 , respectively. The frequency response of the $\frac{1}{4}$ in. microphone is $10 \mathrm{~Hz}-40 \mathrm{kHz}$ so the measurement results below $10 \mathrm{~Hz}$ are not accurate and not shown in Fig. 5. The frequency response of the $\frac{1}{2}$ in. microphone is $2.8 \mathrm{~Hz}-20 \mathrm{kHz}$, therefore the measurement results with the $\frac{1}{2}$ in. microphone are assumed to be accurate from 2.8 to $10 \mathrm{~Hz}$. In the frequency range above $10 \mathrm{~Hz}$, the pressure spectrum measured 


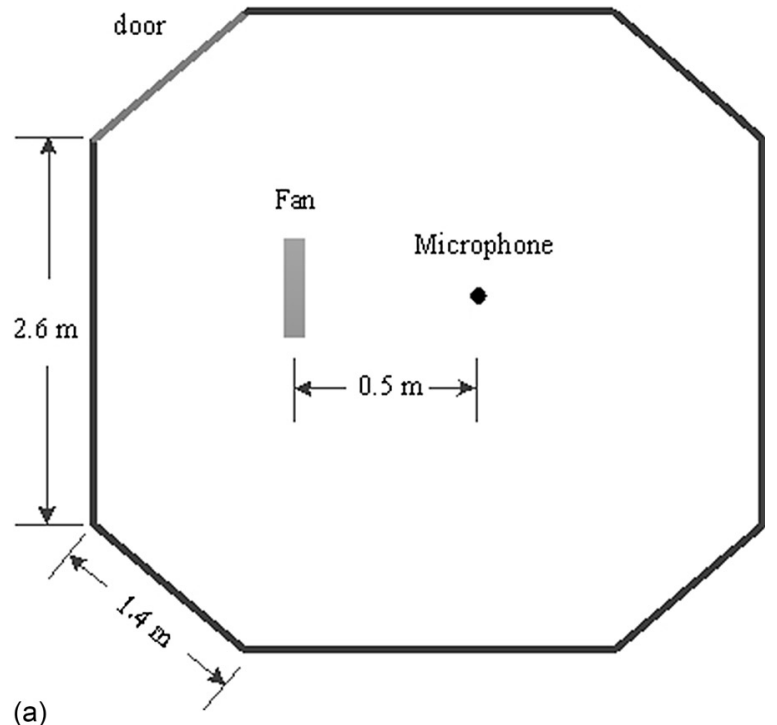

(a)

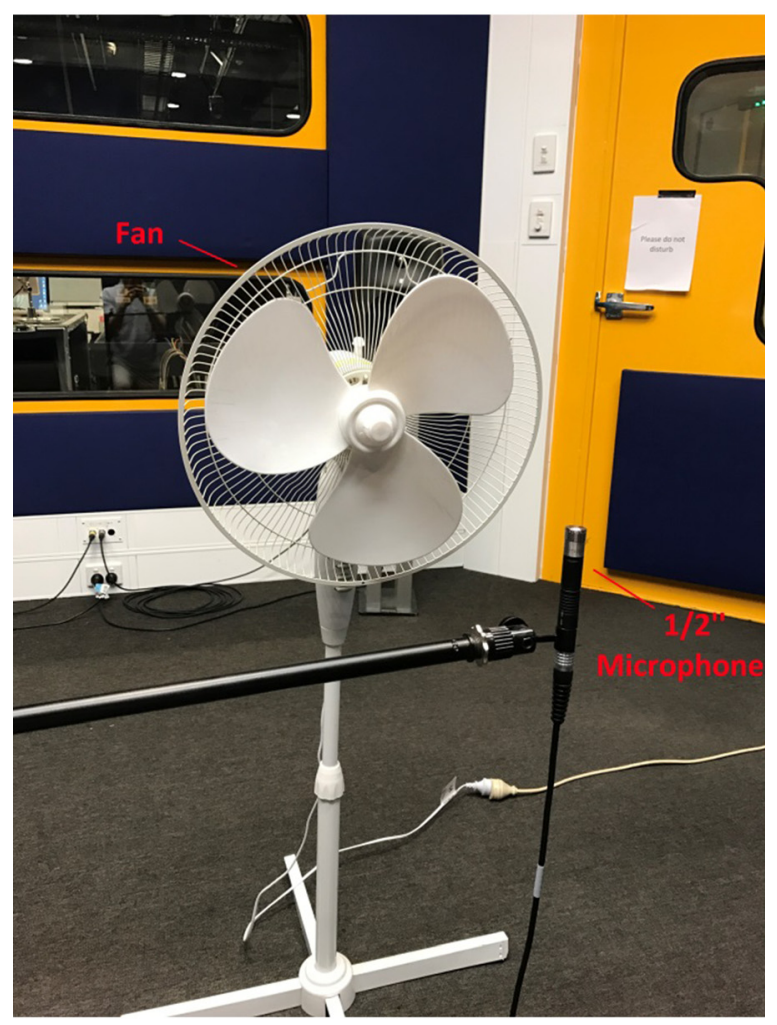

(b)

FIG. 3. (Color online) (a) The diagram and (b) the photo of the experimental setup.

with the $\frac{1}{2}$ in. microphone deviates from that measured with the $\frac{1}{4}$ in. microphone due to the interaction of the microphone with the air flow. The presence of the microphone has two effects on the measured pressure spectrum of the turbulent flow. The first is the wake generated behind the microphone (Strasberg, 1988) and the second is the averaging effect due to the finite size of the microphone diaphragm (Corcos, 1963).

The wake generated by the microphone is usually much smaller than the intrinsic turbulence in the incoming flow, hence it can be neglected according to Morgan and Raspet (1992). To confirm this claim, the wind noise was measured

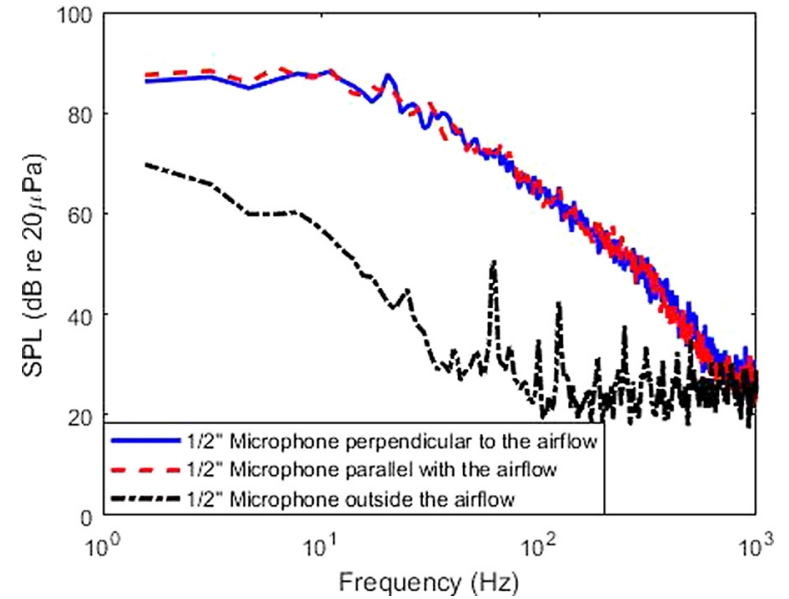

FIG. 4. (Color online) Comparison of the measurement results with the $\frac{1}{2}$ in. microphone perpendicular and parallel to the air flow direction where the black dash-dot line denotes the mechanical noise of the fan with the microphone placed outside of the air flow.

with the $\frac{1}{2}$ in. microphone parallel to the air flow direction so that the wake was far from the diaphragm and had little influence on the measured wind noise spectrum. The measurement results with the $\frac{1}{2}$ in. microphone parallel to and perpendicular with the airflow direction are compared in
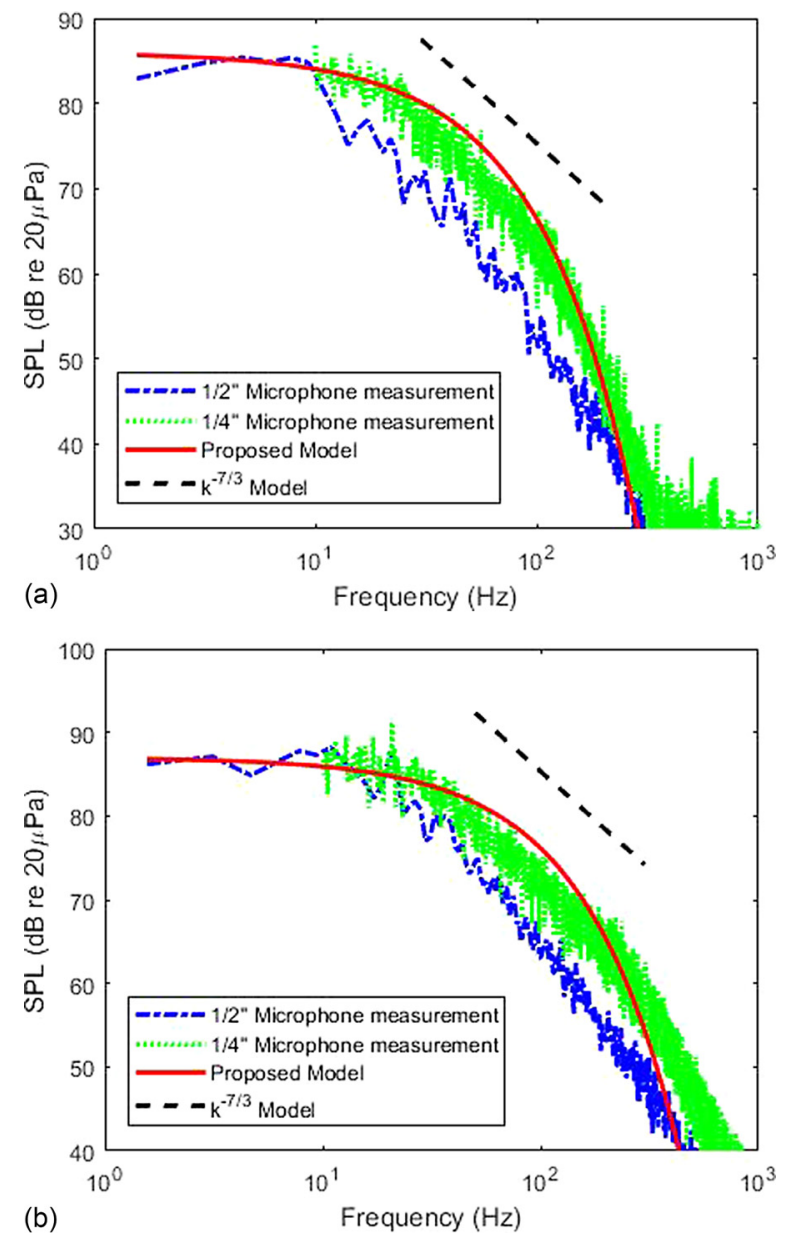

FIG. 5. (Color online) Comparison of the obtained pressure spectrum in Eq. (7) with the indoor fan test results with $\mathrm{a} \frac{1}{2}$ in. microphone and a $\frac{1}{4}$ in. microphone at (a) $U=1.0 \mathrm{~m} / \mathrm{s}\left(\operatorname{Re}_{\lambda} \approx 210\right)$ and (b) $U=3.8 \mathrm{~m} / \mathrm{s}\left(\operatorname{Re}_{\lambda} \approx 410\right)$. 
Fig. 4, which shows that the measurement results were almost the same, hence we can conclude that the wake is negligible compared with turbulence in the incoming flow. In contrast, the averaging effect of the finite size of the microphone diaphragm can introduce undesirable errors in the measurements, especially in the higher frequency range with small eddies (Corcos, 1963). Therefore, the measurement results with the $\frac{1}{4}$ in. microphone are considered to be more accurate than those from the $\frac{1}{2}$ in. microphone in the higher frequency range above $10 \mathrm{~Hz}$.

It can be observed from Fig. 5 that the pressure spectrum obtained from the proposed pressure structure function model agrees with the wind noise spectra measured with the $\frac{1}{2}$ in. microphone below $10 \mathrm{~Hz}$ and that measured with the $\frac{1}{4}$ in. microphone above $10 \mathrm{~Hz}$, which is reasonable according to the above discussions. In contrast, the conventional $k^{-7 / 3}$ model fails to predict the wind noise spectra, especially in the lower frequency range. It is noteworthy that the calculation of the exact values of the constants $r_{\mathrm{L}}$ and $A$ in Eq. (7) needs accurate measurements of the longitudinal velocity at two spatial locations with various separation distances, which requires two channel hot wire anemometers. However, no such hot wire equipment is available to us at the present, so the longitudinal velocity cannot be obtained. In Fig. 5 the proposed model is fitted to the measured wind noise spectra with $r_{\mathrm{L}}=1.67 \times 10^{-2}$ and $A=4.0 \times 10^{5}$.

It is worth noting that the wind noise spectrum measured with the $\frac{1}{4}$ in. microphone in Fig. 5 shows an inertial range with the $k^{-7 / 3}$ law: $30-100 \mathrm{~Hz}$ in Fig. $5($ a) and $50-300 \mathrm{~Hz}$ in Fig. 5(b). This is because the frequency range of the inertial range with the $k^{-7 / 3}$ law depends on the Reynolds number. When the Reynolds number is very small, the inertial range is very small and even vanishes so that it cannot be observed in the pressure spectrum, such as the pressure spectrum in Fig. 2 where the Taylor microscale Reynolds number $\operatorname{Re}_{l}$ is less than 77. This is the ideal case that can match the proposed pressure spectrum model. As the Reynolds number increases, the inertial range extends to a larger range which is observable in the pressure spectrum, the frequency range of the $k^{-7 / 3}$ law increases with the Reynolds number, such as the wind noise spectrum in Fig. 5 where the Taylor microscale Reynolds number is about 210 or 410 , respectively. When the Reynolds number is such large as that in the atmospheric turbulence where the Taylor microscale Reynolds number is over 4250 , the inertial range is so large that the pressure spectrum becomes dominant by the $k^{-7 / 3}$ law (Raspet et al., 2006).

A good wind noise spectrum model should include all the energy-containing range, the inertial range, and the dissipation range in the pressure spectrum. Unfortunately, the mathematical derivation becomes too complicated to obtain an explicit expression of the pressure spectrum if the pressure structure functions of all three ranges from Eq. (2) to Eq. (4) are combined into a single function and substituted to the integral equation in Eq. (5). Because of this difficulty and for the sake of simplicity, the inertial range is omitted in the proposed pressure structure function model in Eq. (6), so that an analytical form of the pressure spectrum could be obtained as Eq. (7). Although the effect of finite Reynolds number is not accounted for in this model, it provides an explanation that the pressure spectrum in small Reynolds number turbulent flows approaches a constant in the lower frequency range and decays rapidly in the higher frequency range, which cannot be deduced from the conventional $k^{-7 / 3}$ model. The quantitative relationship between the finite Reynolds number and the frequency range with the $k^{-7 / 3}$ law in the pressure spectrum needs numerical integral of Eq. (5) and detailed measurements of wind noise spectra in turbulent flows with controlled Reynolds numbers, which will be investigated in the future.

\section{CONCLUSIONS}

This paper proposes a pressure structure function model that combines the energy-containing and dissipation ranges, based on which the pressure spectra can be obtained for small Reynolds number turbulent flows where the inertial range is absent. The results show that the pressure spectra approach a constant in the lower frequency range in the energy-containing range but decay rapidly in the higher frequency range for the dissipation range. The proposed pressure structure function model and the obtained pressure spectra have been validated with both existing numerical and experimental results in the literature as well as indoor fan test measurement results. The pressure spectra obtained from the proposed pressure structure function model can be utilized to predict wind noise measured in indoor environments such as that from fans and wind tunnels. Future work will investigate the effect of the Reynolds number and the presence of microphone on the pressure spectrum.

\section{ACKNOWLEDGMENT}

This research was supported under Australian Research Council's Linkage Projects funding scheme (LP140100740).

Alamshah, V., Zander, A., and Lenchine, V. (2015). "Effects of turbulent flow characteristics on wind induced noise generation in shielded microphones," in Proc. Acoust. 2015, Hunter Valley, Australia, pp. 1-11.

Batchelor, G. K. (1951). "Pressure fluctuations in isotropic turbulence," Math. Proc. Cambridge Philos. Soc. 47, 359-374.

Corcos, G. M. (1963). "Resolution of pressure in turbulence," J. Acoust. Soc. Am. 35, 192-198.

George, W. K., Beuther, P. D., and Arndt, R. E. A. (1984). "Pressure spectra in turbulent free shear flows," J. Fluid Mech. 148, 155-191.

Gotoh, T., and Fukayama, D. (2001). "Pressure spectrum in homogeneous turbulence," Phys. Rev. Lett. 86, 3775-3778.

Hill, D. J., and Wilczak, J. M. (1995). "Pressure structure functions and spectra for locally isotropic turbulence," J. Fluid Mech. 296, 241-269.

Kim, J., and Antonia, R. A. (1993). "Isotropy of the small scales of turbulence at low Reynolds number," J. Fluid Mech. 251, 219-238.

Lohse, D., and Muller-Groeling, A. (1995). "Bottleneck effects in turbulence: Scaling phenomena in $r$ versus $p$ space," Phys. Rev. Lett. 74, $1747-1750$.

Meldi, M., and Sagaut, P. (2013). "Pressure statistics in self-similar freely decaying isotropic turbulence," J. Fluid Mech. 717, 1-12.

Morgan, S., and Raspet, R. (1992). "Investigation of the mechanisms of low-frequency wind noise generation outdoors," J. Acoust. Soc. Am. 92, $1180-1183$.

Ould-Rouis, M., Antonia, R., Zhu, Y., and Anselmet, F. (1996). "Turbulent pressure structure function,” Phys. Rev. Lett. 77, 2222-2224.

Pearson, B. R., and Antonia, R. A. (2001). "Reynolds-number dependence of turbulent velocity and pressure increments," J. Fluid Mech. 444, 343-382.

Pope, S. B. (2000). Turbulent Flows (Cambridge University Press, New York), $771 \mathrm{pp}$. 
Pumir, A. (1994). "A numerical study of pressure fluctuations in threedimensional incompressible, homogeneous, isotropic turbulence," Phys. Fluids 6, 2071-2083.

Raspet, R., and Webster, J. (2015). "Wind noise under a pine tree canopy," J. Acoust. Soc. Am. 137, 651-659.

Raspet, R., Webster, J., and Dillion, K. (2006). "Framework for wind noise studies," J. Acoust. Soc. Am. 119, 834-843.

Raspet, R., Yu, J., and Webster, J. (2008). "Low frequency wind noise contributions in measurement microphones," J. Acoust. Soc. Am. 123, 1260-1269.

Strasberg, M. (1988). "Dimensional analysis of windscreen noise," J. Acoust. Soc. Am. 83, 544-548.
Tatarski, V. I. (1961). Wave Propagation in a Turbulent Medium (Dover, New York), 285 pp.

Tsuji, Y., and Ishihara, T. (2003). "Similarity scaling of pressure fluctuation in turbulence," Phys. Rev. E 68, 026309.

Wang, L., Zander, A. C., and Lenchine, V. V. (2012). "Measurement of the self-noise of microphone wind shields," in Proc. 18th Aust. Fluid Mech. Conf., Launceston, Australia, pp. 1-10.

Webster, J., and Raspet, R. (2015). "Infrasonic wind noise under a deciduous tree canopy," J. Acoust. Soc. Am. 137, 2670-2677.

Zhao, S., Cheng, E., Qiu, X., Burnett, I., and Liu, C. J. (2016). "Pressure spectra in turbulent flows in the inertial and the dissipation ranges," J. Acoust. Soc. Am. 140, 4178-4182. 